\title{
Strong Dominating Sets of Direct Product Graph of Cayley Graphs with Arithmetic Graphs
}

\author{
M. Manjuri \\ Department of Applied Mathematics, \\ Sri Padmavati Women's University, \\ Tirupati, Andhra Pradesh, India.
}

\author{
B. Maheswari \\ Department of Applied Mathematics, \\ Sri Padmavati Women's University, \\ Tirupati, Andhra Pradesh, India.
}

\begin{abstract}
Today, graph theory is one of the most flourishing branches of modern mathematics with wide applications to combinatorial problems and to classical algebraic problems. Graph theory has applications in diverse areas such as social sciences, linguistics, physical sciences, communication engine ering etc. Because of this diversity in applications it is useful to develop and study this subject in abstract terms of the objects of any particular system in which one may be interested.

Product of graphs are introduced in Graph Theory very recently and developing rapidly. In this paper, we consider direct product graphs of Cayley graphs with Arithmetic graphs and discuss strong domination parameter of these graphs.
\end{abstract}

\section{Keywords}

Euler totient Cayley graph, Arithmetic $V_{n}$ graph, direct produ ct graph, Strong dominating set.

\section{Subject Classification: 68R10 \\ 1. INTRODUCTION}

'Domination in graphs' is the fast growing area in Graph Theory that has emerged rapidly in the last four decades. Domination in graphs has applications to several fields such as facility location problems, School Bus Routing, Computer Communication Networks, Radio Stations, Locating Radar Stations Problem etc.,

Number Theory is one of the oldest branches of mathematics, which inherited rich contributions from almost all great mathematicians, ancient and modern.

Nathanson [3] was the pioneer in introducing the concepts of Number Theory, particularly, the 'Theory of Congruences' in Graph Theory, and paved the way for the emergence of a new class of graphs, namely "Arithmetic Graphs". Cayley Graphs are another class of graphs associated with elements of a group. If this group is associated with some Arithmetic function then the Cayley graph becomes an Arithmetic graph. The Cayley graph associated with Euler totient function is called an Euler totient Cayley graph.

Products are often viewed as a convenient language with which one can describe structures, but they are increasingly being applied in more substantial ways. Computer Science is one of the many fields in which graph products are becoming common place.

The direct product was introduced by Alfred North Whitehead and Bertrand Russell in their Principia Mathematica [6] is also called as the tensor product, categorical product, cardinal product, relational product, Kronecker product, weak direct product or conjunction.

\section{Direct Product Graph $G_{1} \times G_{2}$}

Let $G_{1}$ and $G_{2}$ be two simple graphs with their vertex sets as $V_{1}=\left\{u_{1}, \ldots, u_{l}\right\}$ and $V_{2}=\left\{v_{1}, v_{2}, \ldots, v_{m}\right\}$ respectively. Then the direct product of these two graphs denoted by $G_{1} \times G_{2}$ is defined as the graph with vertex set $V_{1} \times V_{2}$, where $V_{1} \times V_{2}$ is the Cartesian product of the sets $V_{1}$ and $V_{2}$ such that any two distinct vertices $\left(u_{1}, v_{1}\right)$ and $\left(u_{2}, v_{2}\right)$ of $G_{1} \times G_{2}$ are adjacent if $u_{1} u_{2}$ is an edge of $G_{1}$ and $v_{1} v_{2}$ is an edge of $G_{2}$.

\section{EULER TOTIENT CAYLEY GRAPH}

For any positive integer $n$, let $Z_{n}=\{0,1,2, ., n-1\}$.

Then $\left(Z_{n}, \oplus\right)$, where $\oplus$ is addition modulo $n$, is an abelian group of order $n$. For any positive integer $n$, let $S$ denote the set of all positive integers less than $n$ and relatively prime to $n$. That is $S=\{r / 1 \leq r<n$ and $\operatorname{GCD}(r, n)=1\}$. Then $|S|=\varphi(n)$, where $\varphi$ is the Euler totient function. We can see that $S$ is a symmetric subset of the group $\left(Z_{n}, \oplus\right)$.

The definition of Euler totient Cayley graph is as follows.

The Euler totient Cayley graph $G\left(Z_{n}, \varphi\right)$ is defined as the graph whose vertex set $V$ is given by $Z_{n}=\{0,1, ., n-1\}$ and the edge set is $E=\{(x, y) / x-y \in S$ or $y-x \in S\}$.

Some properties of Euler totient Cayley graphs and enumeration of Hamilton cycles and triangles can be found in Madhavi [1]. The Euler totient Cayley graph $G\left(Z_{n}, \varphi\right)$ is a complete graph if $n$ is a prime and it is $\varphi(n)$-regular.

The strong domination parameter of these graphs are studied by the authors [2] and the following results are required and they are presented without proofs.

Theorem 2.1: If $n$ is a prime, then the strong domination number of $G\left(Z_{n}, \varphi\right)$ is 1 .

Theorem 2.2: The strong domination number of $G\left(Z_{n}, \varphi\right)$ is 2 , if $n=2 p$ where $p$ is an odd prime.

Theorem 2.3: Suppose $n$ is neither a prime nor $2 p$. Let $n=p_{1}^{\alpha_{1}} p_{2}^{\alpha_{2}} \ldots \ldots p_{k}^{\alpha_{k}}$, where $p_{1}, p_{2}, \ldots p_{k}$ are primes and $\alpha_{1}, \alpha_{2}, \ldots . \alpha_{k}$ are integers $\geq 1$. Then the strong domination number of $G\left(Z_{n}, \varphi\right)$ is given by $\gamma_{s}\left(G\left(Z_{n}, \varphi\right)\right)=\lambda+1$, where $\lambda$ is the length of the longest stretch of consecutive integers in $V$, each of which shares a prime factor with $n$.

\section{ARITHMETIC $\boldsymbol{V}_{\boldsymbol{n}}$ GRAPH}

Let $n$ be a positive integer such that

$n=p_{1}^{\alpha_{1}} p_{2}^{\alpha_{2}} \ldots \ldots p_{k}^{\alpha_{k}}$. Then the Arithmetic $V_{n}$ graph is defined as the graph whose vertex set consists of the divisors of $n$ and two vertices $u$ and $v$ are adjacent in $V_{n}$ graph if and only if $\operatorname{GCD}(u, v)=p_{i}$, for some prime divisor $p_{i}$ of $n$. In this graph vertex 1 becomes an isolated vertex. 
Hence we consider the $V_{n}$ graph without vertex 1 , as the contribution of this isolated vertex is nothing when we study the domination parameters.

Clearly, $V_{n}$ graph is a connected graph. If $n$ is a prime, then $V_{n}$ graph consists of a single vertex. Hence it is connected. In other cases, by the definition of adjacency in $V_{n}$, there exist edges between prime numbers, their prime powers and also to their prime products. Therefore each vertex of $V_{n}$ is connected to some vertex in $V_{n}$.

The strong domination number of $V_{n}$ graph is obtained by the authors and the proof of the following theorem can be found in [2].

Theorem 3.1: If $n=p_{1}^{\alpha_{1}} p_{2}^{\alpha_{2}} . . p_{k}^{\alpha_{k}}$, where $\quad p_{1}, p_{2}, \ldots, p_{k}$ are primes and $\alpha_{1}, \alpha_{2}, \ldots, \alpha_{k}$ are integers $\geq 1$, then the strong domination number of $G\left(V_{n}\right)$ is given by

$$
\gamma_{s}\left(G\left(V_{n}\right)\right)=\left\{\begin{array}{cc}
k-1 & \text { if } \alpha_{\mathrm{i}}=1 \text { for more than one } \mathrm{i}, \\
k & \text { otherwise }
\end{array}\right.
$$

where $k$ is the core of $n$.

\section{DIRECT PRODUCT GRAPH OF $G\left(Z_{n}, \varphi\right)$ WITH $G\left(V_{n}\right)$}

In this paper the direct product graph of Euler totient Cayley graph with Arithmetic $V_{n}$ graph is considered. The properties and some domination parameters of these graphs can be found in [5].

Let $G\left(Z_{n}, \varphi\right)$ denote the Euler Totient Cayley graph and $G\left(V_{n}\right)$ denote the Arithmetic $V_{n}$ graph. Since $G\left(Z_{n}, \varphi\right)$ and $G\left(V_{n}\right)$ are two simple graphs, by the definition of adjacency in the direct product, the graph $G_{1} \times G_{2}$ is a simple graph.

Further the graph $G_{1} \times G_{2}$ is a completely disconnected graph, if $n$ is a prime and the degree of a vertex in $G_{1} \times G_{2}$ is given by

$$
\begin{array}{r}
\operatorname{deg}_{G_{1} \times G_{2}}\left(u_{i}, v_{j}\right)=\operatorname{deg}_{G_{1}}\left(u_{i}\right) \cdot \operatorname{deg}_{G_{2}}\left(v_{j}\right) \\
=\varphi(n) \cdot \operatorname{deg}_{G_{2}}\left(v_{j}\right) .
\end{array}
$$

\section{STRONG DOMINATING SETS OF DIRECT PRODUCT GRAPH}

In this section minimum strong dominating sets of direct product graph of $G\left(Z_{n}, \varphi\right)$ with $G\left(V_{n}\right)$ are discussed and obtained its strong domination number in various cases.

\section{Strong domination}

Let $G(V, E)$ be a graph and $u, v \in V$. Then, $u$ strongly dominates $v$ if (i) $u v \in E$ and (ii) $\operatorname{deg} u \geq \operatorname{deg} v$. A set $D \subset V$ is called a strong dominating set of $G$ if every vertex in $V-D$ is strongly dominated by at least one vertex in $D$.

The strong domination number $\gamma_{S}$ of $G$ is the minimum cardinality of a strong dominating set.

Some results on strong domination for general graphs can be seen in [4].

The results on strong domination of direct product graph $G_{1} \times G_{2}$ are as follows.

Theorem 5.1: If $n$ is a prime, then the strong domination number does not exist for the graph $G_{1} \times G_{2}$.

Proof: Let $n=p$. Then the graph $G_{1} \times G_{2}$ is a completely disconnected graph on $n$ vertices. So, all these vertices form a dominating set. But the given graph does not satisfy the conditions of a strong dominating set.

Therefore the strong domination number does not exist for the graph $G_{1} \times G_{2}$.

Theorem 5.2: If $n=p_{1} p_{2}$, where $p_{1}, p_{2}$ are distinct primes, then the strong domination number of $G_{1} \times G_{2}$ is $n$.

Proof: Let $n=p_{1} p_{2}$, where $p_{1}, p_{2}$ are distinct primes. Consider the graph $G_{1} \times G_{2}$.

Let $V\left(G_{1}\right)=\{0,1, ., n-1\}=V_{1}, V\left(G_{2}\right)=\left\{p_{1}, p_{2}, p_{1} p_{2}\right\}=V_{2}$ and $V\left(G_{1} \times G_{2}\right)=V_{1} \times V_{2}=V$ be the vertex sets of the graphs $G_{1}, G_{2}$ and $G_{1} \times G_{2}$ respectively.

Consider the set

$D=\left\{\left(u_{d_{1}}, p_{1} p_{2}\right),\left(u_{d_{2}}, p_{1} p_{2}\right), \ldots \ldots .,\left(u_{d_{n}}, p_{1} p_{2}\right)\right\}$.

We now prove that $D$ is a dominating set of $G_{1} \times G_{2}$. Consider the vertices in $V-D$ which are $\left\{u_{d_{1}}, u_{d_{2}}, \ldots, u_{d_{n}}\right\} \times$ $\left\{p_{1}, p_{2}\right\}$. Hence the vertices $u_{d_{1}}, u_{d_{2}}, \ldots, u_{d_{n}}$ are consecutive integers in $G_{1}$. So, their difference is 1 , which is in the set $S$ in $G_{1}$, and hence they are adjacent.

Thus the vertices in $V-D$ are adjacent with the vertices $\left\{\left(u_{d_{2}}, p_{1} p_{2}\right), \ldots,\left(u_{d_{n}}, p_{1} p_{2}\right),\left(u_{d_{1}}, p_{1} p_{2}\right)\right\} \quad$ respectively as $\operatorname{GCD}\left(p_{1}, p_{1} p_{2}\right)=p_{1}, G C D\left(p_{2}, p_{1} p_{2}\right)=p_{2}$.

Hence $D$ becomes a dominating set of $G_{1} \times G_{2}$.

Now we check whether $D$ is a strong dominating set or not We know from the properties of Euler totient Cayley graph that $G\left(Z_{n}, \varphi\right)$ is $\varphi(n)$ - regular, where $\varphi(n)$ is the cardinality of the set S. So the degree of each vertex in $G\left(Z_{n}, \varphi\right)$ is $|S|$. For $n=p_{1} p_{2}$, the graph $G_{2}$ contains the vertices $\left\{p_{1}, p_{2}, p_{1} p_{2}\right\}$ and $\operatorname{deg}_{\mathrm{G}_{2}}\left(p_{1} p_{2}\right)=2$,

$\operatorname{deg}_{\mathrm{G}_{2}}\left(p_{1}\right)=1, \operatorname{deg}_{\mathrm{G}_{2}}\left(p_{2}\right)=1$ as there is an edge between $p_{1}$ and $p_{1} p_{2}, p_{2}$ and $p_{1} p_{2}$ and there is no edge between $p_{1}$ and $p_{2}$.

If $\left(u_{i}, v_{j}\right)$ is any vertex of $G_{1} \times G_{2}$, then we know that

$$
\begin{aligned}
\operatorname{deg}_{G_{1} \times G_{2}}\left(u_{i}, v_{j}\right)= & \operatorname{deg}_{G_{1}}\left(u_{i}\right) \cdot \operatorname{deg}_{G_{2}}\left(v_{j}\right) \\
& =\varphi(n) \cdot \operatorname{deg}_{G_{2}}\left(v_{j}\right) .
\end{aligned}
$$

From this, we have

$$
\begin{array}{r}
\operatorname{deg}_{G_{1} \times G_{2}}\left(u_{d_{i}}, p_{1} p_{2}\right)=\operatorname{deg}_{G_{1}}\left(u_{d_{i}}\right) \cdot \operatorname{deg}_{G_{2}}\left(p_{1} p_{2}\right) \\
=\varphi(n) \cdot 2=2 \varphi(n) \\
\operatorname{deg}_{G_{1} \times G_{2}}\left(u_{d_{i}}, p_{1}\right)=\operatorname{deg}_{G_{1}}\left(u_{d_{i}}\right) \cdot \operatorname{deg}_{G_{2}}\left(p_{1}\right) \\
=\varphi(n) .1=\varphi(n) \\
\operatorname{deg}_{G_{1} \times G_{2}}\left(u_{d_{i}}, p_{2}\right)=\operatorname{deg}_{G_{1}}\left(u_{d_{i}}\right) \cdot \operatorname{deg}_{G_{2}}\left(p_{2}\right) \\
=\varphi(n) .1=\varphi(n)
\end{array}
$$

That is $\operatorname{deg}_{G_{1} \times G_{2}}\left(u_{d_{i}}, p_{1} p_{2}\right)>\operatorname{deg}_{G_{1} \times G_{2}}\left(u_{d_{i}}, p_{1}\right)$ and $\operatorname{deg}_{G_{1} \times G_{2}}\left(u_{d_{i}}, p_{2}\right)$-------- (1)

By equation (1), we see that every vertex in $V-D$ is strongly dominated by some vertices in $D$.

Therefore $D$ is a strong dominating set of $G_{1} \times G_{2}$.

Now we show that $D$ is minimal. Suppose we delete a vertex say, $\left(u_{d_{i}}, p_{1} p_{2}\right)$ from $D$. Then we see that $D$ does not form a dominating set of $G_{1} \times G_{2}$, because the vertex $\left(u_{d_{i}}, p_{1} p_{2}\right)$ is not adjacent with any vertex $\left(u_{d_{j}}, p_{1} p_{2}\right), i \neq j$ in $D$ by the 
definition of direct product. Similar is the case by the deletion of any other vertex in $D$. In similar lines we cannot get any strong dominating set minimal than the set $D$.

Therefore $D$ is a minimal strong dominating set of $G_{1} \times G_{2}$ with cardinality $n$.

$$
\text { Hence } \gamma_{S}\left(G_{1} \times G_{2}\right)=n \text {. }
$$

Theorem 5.3: If $n=p^{2}$, then the strong domination number of $G_{1} \times G_{2}$ is 4 .

Proof: Let $n=p^{2}$. Consider the graph $G_{1} \times G_{2}$.

Let $V\left(G_{1}\right)=\left\{0,1,2,3, . . p^{2}-1\right\}=V_{1}, V\left(G_{2}\right)=\left\{p, p^{2}\right\}=V_{2}$ and $V\left(G_{1} \times G_{2}\right)=V_{1} \times V_{2}=V$, be the vertex sets of $G_{1}, G_{2}$ and $G_{1} \times G_{2}$ respectively.

By the properties of Euler totient Cayley graph, the graph $G\left(Z_{n}, \varphi\right)$ is $\varphi(n)$ - regular. So, each vertex in $G\left(Z_{n}, \varphi\right)$ has same degree $\varphi(n)$. For $n=p^{2}$, the graph $G\left(Z_{n}, \varphi\right)$ is $\left(p^{2}-p\right)-$ regular and hence each vertex has degree $p^{2}-p$. The graph $G_{2}$ contains two vertices $p$ and $p^{2}$ and these two vertices are joined by an edge as $G C D\left(p, p^{2}\right)=p$.

By the properties of direct product, the graph $G_{1} \times G_{2}$ is $p(p-1)-$ regular, so that the degree of every vertex in $G_{1} \times G_{2}$ is $p(p-1)$.

$$
\text { Let } D=\left\{\left(u_{d_{1}}, p\right),\left(u_{d_{1}}, p^{2}\right),\left(u_{d_{2}}, p\right),\left(u_{d_{2}}, p^{2}\right)\right\} \text {. }
$$

Now we show that $D$ is a dominating set of $G_{1} \times G_{2}$. Let $(u, v)$ be any vertex of $V-D$ in $G_{1} \times G_{2}$. Then the vertex $u$ in $G_{1}$ is adjacent with either $u_{d_{1}}$ or $u_{d_{2}}$ as $D_{1}$ is a dominating set of $G_{1}$. The vertices $p, p^{2}$ in $G_{2}$ are adjacent as $G C D\left(p, p^{2}\right)=p$.

Thus by the definition of direct product a vertex $(u, v)$ in $V-$ $D$ is adjacent with either $\left(u_{d_{1}}, p\right)$ or $\left(u_{d_{1}}, p^{2}\right)$;

or $\left(u_{d_{2}}, p\right)$ or $\left(u_{d_{2}}, p^{2}\right)$ in $D$.

Therefore $D$ is a dominating set and it also becomes a strong dominating set of $G_{1} \times G_{2}$ as the graph is regular.

Now we show that $D$ is minimal. That is deletion of any vertex in $D$ does not make $D$, a dominating set any more.

Suppose the vertex $\left(u_{d_{1}}, p\right)$ is deleted from $D$. We know that the degree of a vertex in $G_{1}$ is $\varphi(n)$. So let $u_{d_{1}}$ be adjacent with the vertices $u_{1}, u_{2}, \ldots . u_{\varphi(n)}$ in $G_{1}$. Consider the set of vertices $S=\left\{\left(u_{1}, p^{2}\right),\left(u_{2}, p^{2}\right), \ldots,\left(u_{\varphi(n)}, p^{2}\right)\right\}$ in $G_{1} \times G_{2}$. Since $\operatorname{deg}_{G_{1}}\left(u_{d_{2}}\right)$ is $\varphi(n)$, all the vertices $u_{1}, u_{2}, \ldots \ldots, u_{\varphi(n)}$ are not dominated by $u_{d_{2}}$. Otherwise the vertices of $V_{1}-$ $\left\{u_{1}, u_{2}, \ldots, u_{\varphi(n)}\right\}$ in $G_{1}$ are dominated neither by $u_{d_{1}}$

nor by $u_{d_{2}}$, because it is a null graph. So all the vertices in $\mathrm{S}$ are not dominated by $\left(u_{d_{2}}, p\right)$ and $\left(u_{d_{2}}, p^{2}\right)$. Since there is no edge between $p^{2}$ to itself in $G_{2}$, all the vertices in $\mathrm{S}$ are not dominated by $\left(u_{d_{1}}, p^{2}\right)$. Thus no vertex in $D-\left\{\left(u_{d_{1}}, p\right)\right\}$ can dominate the vertices of S. Thus $D-\left\{\left(u_{d_{1}}, p\right)\right\}$ is not a dominating set of $G_{1} \times G_{2}$. Similar is the case with the deletion of any other vertex in $D$. Thus $D$ becomes a minimal strong dominating set of $G_{1} \times G_{2}$ with cardinality 4 .

$$
\text { Hence } \gamma_{S}\left(G_{1} \times G_{2}\right)=4 \text {. }
$$

Theorem 5.4: If $n$ is neither a prime nor $p_{1} p_{2}$ nor $p^{2}$ and $n=p_{1}^{\alpha_{1}} p_{2}^{\alpha_{2}} \ldots \ldots p_{k}^{\alpha_{k}}$, where $p_{1}, \ldots, p_{k}$ are distinct primes and $\alpha_{i} \geq 1$, then the strong domination number of $G_{1} \times G_{2}$ is given by

$$
\begin{aligned}
& \gamma_{s}\left(G_{1} \times G_{2}\right) \\
& =\left\{\begin{array}{cc}
n k & \text { if } \alpha_{\mathrm{i}}>1 \text { for all } i, \\
(\lambda+1) k & \text { if } \alpha_{\mathrm{i}}=1 \text { for all } i, \\
n(k-1)+(\lambda+1) & \text { if } \alpha_{\mathrm{i}}=1 \text { for only one } \mathrm{i}, \\
n k_{1}+(\lambda+1) k_{2} & \text { otherwise. }
\end{array}\right.
\end{aligned}
$$

where $\lambda$ is the length of the longest stretch of consecutive integers in $V_{1}$ of $G_{1}$ each of which shares a prime factor with $n, k$ is the core of $n, k_{1}$ is the number of primes with exponent $>1$ and $k_{2}$ is the number of primes with exponent 1 .

Proof: Let $n$ be neither a prime nor $p_{1} p_{2}$ nor $p^{2}$.

Suppose $n=p_{1}^{\alpha_{1}} p_{2}^{\alpha_{2}} \ldots p_{k}^{\alpha_{k}}$, where $\alpha_{i} \geq 1$. Let $V_{1}, V_{2}, V$ denote the vertex sets of $G_{1}, G_{2}$ and $G_{1} \times G_{2}$ respectively. By Theorem 2.3, the strong domination number of $G_{1}$ is $\lambda+1$.

Let $D_{1}=\left\{u_{d_{1}}, \ldots, u_{d_{\lambda+1}}\right\}$ be a strong dominating set of $G_{1}$, where $u_{d_{1}}, u_{d_{2}}, \ldots, u_{d_{\lambda+1}}$ are consecutive integers. Again by Theorem 3.1,

$$
\gamma_{s}\left(G_{2}\right)=\left\{\begin{array}{cc}
k-1 & \text { if } \alpha_{\mathrm{i}}=1 \text { for more than one } \mathrm{i}, \\
k & \text { otherwise. }
\end{array}\right.
$$

where $k$ is the core of $n$.

Now we have the following possibilities.

Case 1: Suppose $\alpha_{i}>1$ for all $i$. By Theorem 3.1 of Case 1, the strong dominating set of $G_{2}$ with minimum cardinality $k$ is given by $D_{2}=\left\{p_{1}, p_{2}, \ldots, p_{k}\right\}$.

That is $\operatorname{deg}\left(p_{i}\right) \geq \operatorname{deg}\left(v_{j}\right)$ for any $v_{j} \in V_{2}-D_{2}$.

Further $D_{1}=\left\{u_{d_{1}}, u_{d_{2}}, \ldots, u_{d_{\lambda+1}}\right\}$ is a dominating set of $G_{1}$.

Consider the set $\quad D=D_{1} \times D_{2}=\left\{u_{d_{1}}, u_{d_{2}}, \ldots, u_{d_{\lambda+1}}\right\} \times$ $\left\{p_{1}, p_{2}, \ldots \ldots, p_{k}\right\}$.

Then a vertex $\left(u_{d_{i}}, p_{j}\right) \in V-D$ where $i=\lambda+2, \ldots, n$;

$j=1,2, \ldots, k$ is not adjacent with any vertex in $D$ as there are no edges between the primes $p_{1}, p_{2}, \ldots \ldots, p_{k}$ in $G_{2}$.

Therefore $D$ can't be a dominating set of $G_{1} \times G_{2}$.

So we have to extend the set $D_{1}$ and let $D_{1}$ be the entire vertex set $V_{1}$ of $G_{1}$.

Let $D=\left\{u_{d_{1}}, u_{d_{2}}, \ldots, u_{d_{n}}\right\} \times\left\{p_{1}, p_{2}, \ldots, p_{k}\right\}$, where the vertices $\left\{u_{d_{1}}, u_{d_{2}}, \ldots, u_{d_{n}}\right\}$ are in consecutive order.

Let $(u, v) \in V-D$. Then $u=u_{d_{i}}$ for some $i$. Since $D_{2}=$ $\left\{p_{1}, p_{2}, \ldots, p_{k}\right\}$ is a dominating set of $G_{2}$, the vertex $v \in V_{2}-$ $D_{2}$ is adjacent with some vertex, say $p_{j}$ in $D_{2}$. Then the vertex $(u, v)=\left(u_{d_{i}}, v\right)$ is adjacent with the vertex $\left(u_{d_{i+1}}, p_{j}\right)$ in $D$.

Hence $D$ becomes a dominating set of $G_{1} \times G_{2}$.We now show that $D$ is a strong dominating set of $G_{1} \times G_{2}$.

$$
\begin{aligned}
& \text { Let }\left(u_{d_{i}}, v_{j}\right) \text { be any vertex in } V-D . \text { Then } \\
& \begin{aligned}
\operatorname{deg}_{G_{1} \times G_{2}}\left(u_{d_{i}}, p_{i}\right) & =\varphi(n) \cdot \operatorname{deg}_{G_{2}}\left(p_{i}\right), \\
& \geq \varphi(n) \operatorname{deg}_{G_{2}}\left(v_{j}\right), \text { where } p_{i} \neq v_{j}, \\
& \geq \operatorname{deg}_{G_{1} \times G_{2}}\left(u_{d_{i}}, v_{j}\right) .
\end{aligned}
\end{aligned}
$$

This implies that every vertex in $V-D$ is strongly dominated by some vertex in $D$. 
Thus $D$ is a strong dominating set of $G_{1} \times G_{2}$.

We now show that $D$ is minimal. Suppose we delete a vertex, say $\left(u_{d_{i}}, p_{j}\right)$ from $D$. Obviously this vertex is not dominated by any vertex in $D$ as $p_{1}, p_{2}, \ldots, p_{k}$ are primes, there is no adjacency between these vertices in $G_{2}$. Therefore $D-$ $\left(u_{d_{i}}, p_{j}\right)$ can't be a dominating set of $G_{1} \times G_{2}$. Similar is the case with the deletion of any other vertex in $D$.

Thus $D$ is a minimal strong dominating set of $G_{1} \times G_{2}$.

Hence $\gamma_{s}\left(G_{1} \times G_{2}\right)=n . k$.

Case 2: Suppose $\alpha_{\mathrm{i}}=1$ for all $i$. That is $n=p_{1} p_{2} \ldots \ldots p_{k}$. By Theorem 3.1 of Case 3, we know that $\gamma_{s}\left(G_{2}\right)=k-1$, where $k$ is the core of $n$ and a strong dominating set in $G_{2}$ is given by $D_{2}=\left\{p_{1}, p_{1} p_{2}, p_{2} p_{3}, \ldots \ldots ., p_{k-3} p_{k-2}, p_{k-1} p_{k}\right\}$.

Further we know that $D_{1}=\left\{u_{d_{1}}, u_{d_{2}}, \ldots, u_{d_{\lambda+1}}\right\}$ is a dominating set of $G_{1}$.

If we consider the set $D=D_{1} \times D_{2}=\left\{u_{d_{1}}, u_{d_{2}}, ., u_{d_{\lambda+1}}\right\} \times$ $\left\{p_{1}, p_{1} p_{2}, p_{2} p_{3}, ., p_{k-3} p_{k-2}, p_{k-1} p_{k}\right\}$, then we can't prove that $D$ is a dominating set of $G_{1} \times G_{2}$, because the vertices $\left(u_{d_{l}}, p_{k-1} p_{k}\right), l=\lambda+2, \lambda+3, \ldots, n$ in $V-D$ are not adjacent with any vertex in $D$, because

$G C D\left(p_{k-1} p_{k}, p_{i} p_{j}\right)=1, i=1,2, \ldots \ldots \ldots, k-3$;

$j=2,3, ., k-2$ and $\operatorname{GCD}\left(p_{k-1} p_{k}, p_{1}\right)=1$.

Therefore we modify the set $D_{2}$ by including a vertex viz., $p_{k-2} p_{k-1}$.

Then $D_{2}=\left\{p_{1}, p_{1} p_{2}, p_{2} p_{3}, \ldots, p_{k-3} p_{k-2}, p_{k-2} p_{k-1}, p_{k-1} p_{k}\right\}$. Obviously $D_{2}$ is a dominating set of $G_{2}$ which is also strong.

Let $D=\left\{u_{d_{1}}, u_{d_{2}}, \ldots, u_{d_{\lambda+1}}\right\} \times\left\{p_{1}, p_{1} p_{2}, p_{2} p_{3}, \ldots, p_{k-3} p_{k-2}\right.$, $\left.p_{k-2} p_{k-1}, p_{k-1} p_{k}\right\}$.

Let $(u, v)$ be any vertex of $V-D$ in $G_{1} \times G_{2}$. Then the vertex $u$ is adjacent with some vertex $u_{d_{l}}$ in $D_{1}$ and vertex $v$ is adjacent with either $p_{1}$ or $p_{i} p_{j}, i=1, \ldots \ldots, k-1$,

$j=2, \ldots . k$, as $D_{1}$ and $D_{2}$ are dominating sets of $G_{1}$ and $G_{2}$ respectively. That is any vertex $(u, v)$ in $V-D$ is adjacent with $\left(u_{d_{l}}, p_{1}\right)$ or $\left(u_{d_{l}}, p_{i} p_{j}\right)$ in $D$. Thus the vertices in $V-D$ are adjacent with at least one vertex in $D$.

Therefore $D$ is a dominating set of $G_{1} \times G_{2}$. We now show that $D$ is a strong dominating set in $G_{1} \times G_{2}$.

Let $\left(u_{d_{i}}, v_{j}\right)$ be any vertex in $V-D$. Then we have

$$
\begin{aligned}
\operatorname{deg}_{G_{1} \times G_{2}}\left(u_{d_{i}}, p_{i} p_{j}\right) & =\varphi(n) \cdot \operatorname{deg}_{G_{2}}\left(p_{i} p_{j}\right), \\
& \geq \varphi(n) \cdot \operatorname{deg}_{G_{2}}\left(v_{j}\right), \\
& \geq \operatorname{deg}_{G_{1} \times G_{2}}\left(u_{d_{i}}, v_{j}\right)
\end{aligned}
$$

and $\operatorname{deg}_{G_{1} \times G_{2}}\left(u_{d_{i}}, p_{1}\right)=\varphi(n) \cdot \operatorname{deg}_{G_{2}}\left(p_{1}\right)$,

$$
\geq \varphi(n) \cdot \operatorname{deg}_{G_{2}}\left(v_{j}\right)
$$

$v_{j}=p_{i}, p_{i} p_{j} p_{l}, \ldots ., p_{i} p_{j} p_{l} . . p_{k}$

$$
\geq \operatorname{deg}_{G_{1} \times G_{2}}\left(u_{d_{i}}, v_{j}\right) \text {. }
$$

This implies that every vertex in $V-D$ is strongly dominated by some vertex in $D$.

Thus $D$ is a strong dominating set of $G_{1} \times G_{2}$.

We now show that $D$ is minimal. Suppose we delete a vertex, say $\left(u_{d_{i}}, p_{1}\right)$ from $D$. Then the vertex $\left(u_{d_{i}}, p_{1}, p_{2}, \ldots, p_{k}\right)$ is not adjacent with any vertex in $D$, because

$\operatorname{GCD}\left(p_{1} p_{2} \ldots p_{k}, p_{i} p_{j}\right)=p_{i} p_{j}$, where $i=1,2, \ldots, k-1$;

$j=2,3, \ldots, k$.

Similar is the case with the deletion of any other vertex in $D$.

Thus $D$ is a minimaml strong dominating set of $G_{1} \times G_{2}$ with cardinality $(\lambda+1) k$.

Hence $\gamma_{s}\left(G_{1} \times G_{2}\right)=(\lambda+1) k$.

Case 3: Suppose $\alpha_{\mathrm{i}}=1$ for only one $i$.

Let $n=p_{1}^{\alpha_{1}} p_{2}^{\alpha_{2}} \ldots p_{i-1}^{\alpha_{i-1}} p_{i} p_{i+1}^{\alpha_{i+1}} \ldots p_{k}^{\alpha_{k}}$.

By Theorem 3.1 of Case 2, a strong dominating set of $G_{2}$ is given by

$\left\{p_{1}, p_{2}, . ., p_{i-1}, p_{i} p_{j}, p_{i+1}, . ., p_{k}\right\}$ and $\operatorname{deg}\left(p_{i} p_{j}\right)>\operatorname{deg}\left(p_{i}\right)$,

$j=1,2, ., i-1, i+1, \ldots, k$.

Let $D=\left\{u_{d_{1}}, u_{d_{2}}, ., u_{d_{n}}\right\} \times\left\{p_{1}, p_{2}, ., p_{i-1}, p_{i+1}, ., p_{k}\right\} \cup$

$\left\{u_{d_{1}}, ., u_{d_{\lambda+1}}\right\} \times\left\{p_{i} p_{j}\right\}$.

Now we show that $D$ is a dominating set of $G_{1} \times G_{2}$, where the vertices $u_{d_{1}}, u_{d_{2}}, \ldots, u_{d_{n}}$ are in consecutive order in $G_{1}$. The vertices $\left\{u_{d_{\lambda+2}}, u_{d_{\lambda+3}}, \ldots, u_{d_{n}}\right\} \times\left\{p_{i} p_{j}\right\}$ in $V-D$ of $G_{1} \times G_{2}$ are dominated by some vertex in $\left\{u_{d_{1}}, u_{d_{2}}, ., u_{d_{n}}\right\} \times$ $\left\{p_{1}, p_{2}, ., p_{i-1}, p_{i+1}, . ., p_{k}\right\} \in \mathrm{D}$, as $G C D\left(p_{i} p_{j}, p_{j}\right)=p_{j}$. So the vertices $\left\{u_{d_{\lambda+2}}, u_{d_{\lambda+3}}, ., u_{d_{n}}\right\} \times\left\{p_{i}\right\}$ in $V-D$ of $G_{1} \times G_{2}$ are dominated by some vertices in $\left\{u_{d_{1}}, u_{d_{2}}, u_{d_{3}}, \ldots, u_{d_{\lambda+1}}\right\} \times$ $\left\{p_{i} p_{j}\right\} \in \mathrm{D}$.

Thus every vertex in $V-D$ is adjacent with at least one vertex in $D$.

Therefore $D$ is dominating set of $G_{1} \times G_{2}$.

Now we show that $D$ is a strong dominating set of $G_{1} \times G_{2}$. Let $\left(u_{d_{i}}, v_{j}\right)$ be any vertex of $V-D$. Then we have

$$
\begin{aligned}
\operatorname{deg}_{G_{1} \times G_{2}}\left(u_{d_{i}}, p_{j}\right) & =\varphi(n) \cdot \operatorname{deg}_{G_{2}}\left(p_{j}\right) \\
& =1,2, \ldots i-1, i+1, \ldots, k \\
& \geq \varphi(n) \cdot \operatorname{deg}_{G_{2}}\left(v_{j}\right) \\
& \geq \operatorname{deg}_{G_{1}}\left(u_{d_{i}}\right) \cdot \operatorname{deg}_{G_{2}}\left(v_{j}\right),
\end{aligned}
$$

where $\left(u_{d_{i}}, v_{j}\right) \in V-D$

$$
\text { and } \operatorname{deg}_{G_{1} \times G_{2}}\left(u_{d_{i}}, p_{i} p_{j}\right) \geq \varphi(n) \cdot \operatorname{deg}_{G_{2}}\left(v_{j}\right), v_{j} \neq p_{j} \text {. }
$$

Thus every vertex in $V-D$ of $G_{1} \times G_{2}$ is strongly dominated by at least one vertex in $D$ of $G_{1} \times G_{2}$.

Therefore $D$ is a strong dominating set of $G_{1} \times G_{2}$.

Now we show that $D$ is minimal. Suppose we delete some vertex, say $\left(u_{d_{i}}, p_{j}\right)$,

$i=1,2, \ldots \ldots, n ; j=1,2, \ldots . i-1, i+1, \ldots \ldots, k$ from $D$. Then we can see that $D$ is no more a dominating set of $G_{1} \times G_{2}$, because the vertex $\left(u_{d_{l}}, p_{j}{ }^{r}\right), r>1$ is adjacent

only with the vertex $\left(u_{d_{i}}, p_{j}\right)$, as $G C D\left(p_{j}^{r}, p_{l}\right)=1$,

$G C D\left(p_{j}^{r}, p_{i} p_{l}\right)=1, j \neq l$ and this vertex is not in $D$.

Thus $D$ is a minimal strong dominating set of $G_{1} \times G_{2}$ with cardinality $n(k-1)+(\lambda+1)$.

Hence $\gamma_{s}\left(G_{1} \times G_{2}\right)=n(k-1)+(\lambda+1)$.

Case 4: Suppose $\alpha_{\mathrm{j}}=1$ for some $j=1,2, \ldots, i$ and $\alpha_{\mathrm{j}}>1$ for $j=i+1, i+2, \ldots, k$. That is $n=p_{1} . p_{2} \ldots \ldots . p_{i} p_{i+1}^{\alpha_{i+1}} \ldots \ldots p_{k}^{\alpha_{k}}$. 
By Theorem 3.1 of Case 3, we know that $\gamma_{s}\left(G_{2}\right)=k-1$ where $k$ is the core of $n$.

Let $D=\left\{u_{d_{1}}, u_{d_{2}}, \ldots ., u_{d_{n}}\right\} \times\left\{p_{i+1}, p_{i+2}, ., p_{k}\right\} \cup$

$\left\{u_{d_{1}}, u_{d_{2}}, \ldots \ldots, u_{d_{\lambda+1}}\right\} \times\left\{p_{k} p_{1}, p_{1} p_{2}, \ldots \ldots \ldots, p_{i-1} p_{i}\right\}$. Then by the above Cases 1 and 2 , it follows that $D$ is a strong dominating set of $G_{1} \times G_{2}$.

We show that $D$ is minimal. If we delete a vertex, say $\left(u_{d_{1}}, p_{i+1}\right)$ from $D$, then $D-\left(u_{d_{1}}, p_{i+1}\right)$ can be no more a dominating set, as there is no vertex in $D-\left(u_{d_{1}}, p_{i+1}\right)$ dominating the vertex $\left(u_{d_{1}}, p_{i+1}\right)$, since $\operatorname{GCD}\left(p_{i+1}, p_{j}\right)=1$, $j=i+2, i+3, \ldots \ldots, k$.

\section{ILLUSTRATIONS}

$n=13($ Prime number $)$

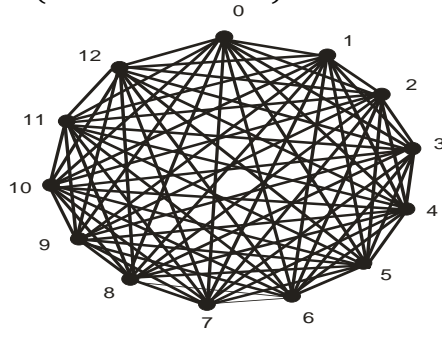

Fig.1

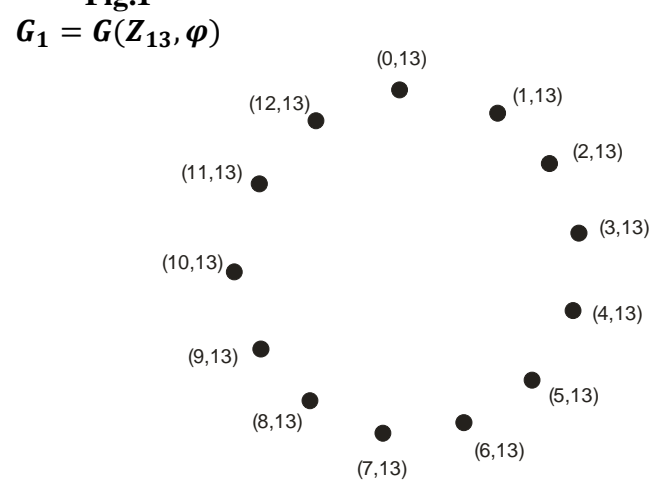

Fig.3

$G_{1} \times G_{2}$

Strong dominating set does not exist
Therefore $D=\left\{u_{d_{1}}, u_{d_{2}}, \ldots, u_{d_{n}}\right\} \times\left\{p_{i+1}, p_{i+2}, . ., p_{k}\right\} \cup$ $\left\{u_{d_{1}}, u_{d_{2}}, \ldots ., u_{d_{\lambda+1}}\right\} \times\left\{p_{k} p_{1}, p_{1} p_{2}, \ldots \ldots \ldots, p_{i-1} p_{i}\right\}$ is a strong dominating set of $G_{1} \times G_{2}$ with minimum cardinality $n k_{1}+(\lambda+1) k_{2}$.

$$
\text { Hence } \gamma_{s}\left(G_{1} \times G_{2}\right)=n k_{1}+(\lambda+1) k_{2} \text {. }
$$

\section{CONCLUSION}

The strong dominating sets of Euler totient Cayley graphs and Arithmetic $V_{n}$ graphs are studied by the authors. This study is motivated to find the strong dominating sets of Direct product graph of Euler totient Cayley graphs with Arithmetic $V_{n}$ graph. Further the Strong dominating sets of strong product graph and Lexicographic product graph of these graphs are also studied.
Fig.2

$G_{2}=G\left(V_{13}\right)$

$n=2 \times 5=10$

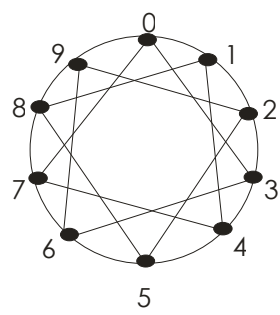

Fig.4

$G_{1}=G\left(Z_{10}, \varphi\right)$

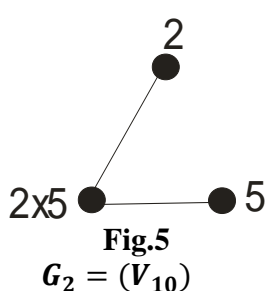

$\boldsymbol{G}_{\mathbf{2}}=\left(\boldsymbol{V}_{\mathbf{1 0}}\right)$ 


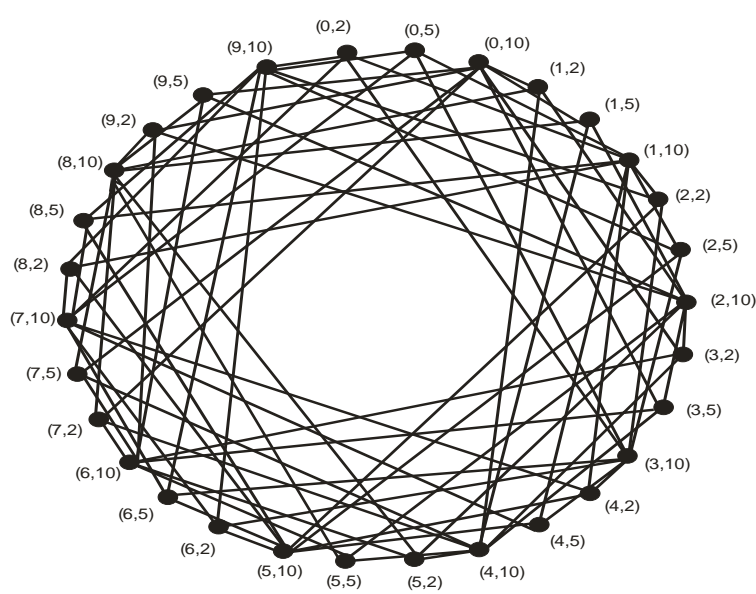

Fig.6

$\boldsymbol{G}_{1} \times \boldsymbol{G}_{2}$

Strong dominating set $\{(0,10),(1,10),(2,10), \ldots,(9,10)\}$ $n=2^{3}=8$

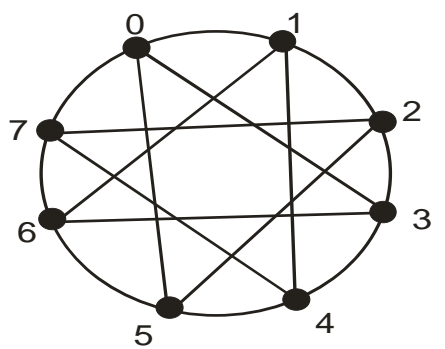

Fig.7

$G_{1}=G\left(Z_{8}, \varphi\right)$

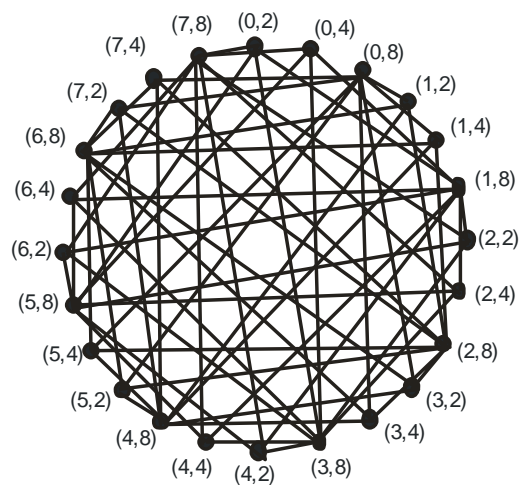

Fig.9

$\boldsymbol{G}_{\mathbf{1}} \times \boldsymbol{G}_{\mathbf{2}}$

Strong dominating set $\{(0,2),(1,2), . .,(7,2)\}$

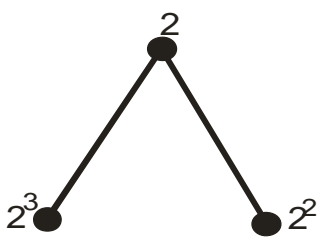

Fig.8

$G_{2}=G\left(V_{8}\right)$

$n=3^{2}=9$

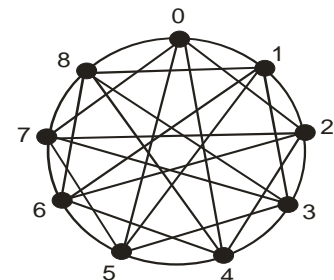

Fig.10

$G_{1}=G\left(Z_{9}, \varphi\right)$

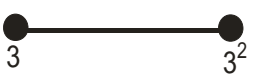

Fig.11

$G_{2}=G\left(V_{9}\right)$ 


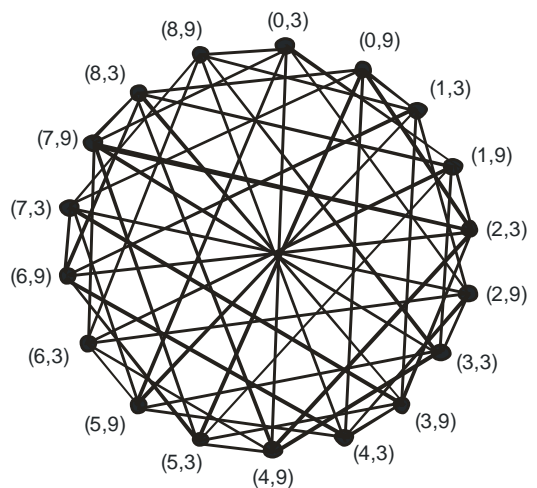

Fig.12

$G_{1} \times G_{2}$ Strong dominating set $\{(0,3),(1,3),(0,9),(1,9)\}$

\section{REFERENCES}

[1] Madhavi, L. - Studies on domination parameters and enumeration of cycles in some Arithmetic graphs, Ph. D. Thesis submitted to S.V.University, Tirupati, India (2002).

[2] Manjuri, M. and Maheswari, B.- Strong dominating sets of Euler totient Cayley graph and Arithmetic Vn graphs, International Journal of Computer Applications (IJCA), Volume 83, No3 (2013), 36-40.

[3] Nathanson and B.Melvyn, -Connected components of arithmetic graphs, Monat.fur.Math, 29, (1980), 219 220 .
[4] Sampathkumar, E. and Pushpa Latha, L.-, Strong weak domination and domination balance in graph, Discrete Mathematics, 161 (1996), 235-242.

[5] Uma Maheswari, S.- Some studies on the product graphs of Euler totient Cayley graphs and Arithmetic Vn graphs, $\mathrm{Ph}$. D. Thesis submitted to S.P.Women's University, Tirupati, India (2012).

[6] Whitehead, A.N., Russel, B.- Principia Mathematica, Volume 2, Cambridge University Press, Cambridge (1912). 